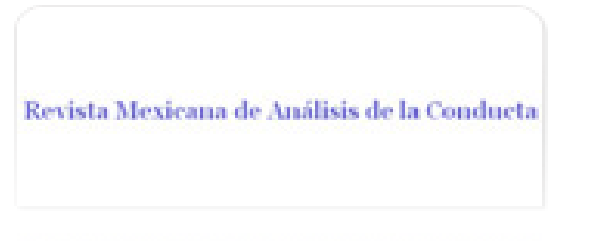

Revista Mexicana de Análisis de la Conducta ISSN: 0185-4534

editora@rmac-mx.org

Sociedad Mexicana de Análisis de la Conducta México

Hernández Castro, Rosendo; Cortés Moreno, Assol

Comportamiento afectivo y complejidad lingüística del infante en díadas madre-hijo Revista Mexicana de Análisis de la Conducta, vol. 35, núm. 1, junio, 2009, pp. 93-111

Sociedad Mexicana de Análisis de la Conducta

Guadalajara, México

Disponible en: http://www.redalyc.org/articulo.oa?id=59311416006

- Cómo citar el artículo

- Número completo

- Más información del artículo

Página de la revista en redalyc.org

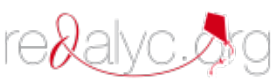

Sistema de Información Científica

Red de Revistas Científicas de América Latina, el Caribe, España y Portugal Proyecto académico sin fines de lucro, desarrollado bajo la iniciativa de acceso abierto 


\title{
COMPORTAMIENTO AFECTIVO Y COMPLEJIDAD LINGÜÍSTICA DEL INFANTE EN DÍADAS MADRE-HIJO
}

\author{
AFFECTIVE BEHAVIOR AND LINGUISTIC COMPLEXITY \\ OF INFANT IN DYADS MOTHER-SON
}

\author{
ROSENDO HERNÁNDEZ CASTRO Y ASSOL CORTÉS MORENO'1 \\ PROYECTO DE INVESTIGACIÓN EN APRENDIZAJE HUMANO (PIAH ) \\ UNAM, FES IZTACALA
}

\begin{abstract}
RESUMEN
El estudio fue observacional y evalúo la relación entre el comportamiento afectivo y la complejidad lingüística del niño en díadas madre-hijo. Los participantes fueron 8 díadas madre-hijo, cuatro con niños de 3 años y cuatro con niños de 5 años de edad. Las díadas fueron video-filmadas en juego libre y juego simulado durante 30 minutos cada uno, en el hogar de los participantes. Fueron codificados 20 minutos de cada sesión para identificar los episodios interactivos de complejidad funcional y conducta afectiva de cada participante. Los resultados muestran que algunas categorías de conducta afectiva positiva se relacionan con la edad del niño, la situación de juego y la complejidad funcional de los participantes. Los datos se discuten en términos de las condiciones en las que es más probable la ocurrencia de la conducta afectiva y su importancia para el comportamiento de mayor complejidad.

Palabras clave: Conducta afectiva, complejidad lingüística, díadas madre-hijo.

1. Los autores agradecen a las madres de familia y los niños por permitirnos entrar a sus hogares y por su participación desinteresada en este estudio. Agradecemos a Lorena Domínguez y Daniel Yañez por su invaluable colaboración en las filmaciones y codificación de los videos, respectivamente. Enviar correspondencia a: Rosendo Hernández Castro, Proyecto de Investigación en Aprendizaje Humano (PIAH), FES Iztacala-UNAM, Av. de los Barrios \#1, Los Reyes Iztacala, Tlalnepantla , Estado de México. CP. 54090. e-mail: rosendohc@correo.unam.mx

Recibido: Noviembre 26, 2008 Revisado: Enero 14, 2009 Aceptado: Mayo 11, 2009
\end{abstract}




\begin{abstract}
This observational study assessed the relation between affective behavior and linguistic complexity in mother-son dyads. The participants were eight dyads mother-son, four children were 3 -years-olds and four 5 -years-old. Dyads were videotaped 30 minutes while they interacted in two conditions, free play and pretend play. For analysis functional complexity and affective behavior were identified, 20 minutes were codified. The results show that some categories of positive affective behavior are related to the children age, play situation, and the child and mother behavioral complexity. The data are discussed in terms of conditions under which it is more likely the occurrence of positive affective behavior and its importance for child behavior complexity.
\end{abstract}

Key Words: Affective behavior, linguistic complexity, adult-child dyads

El efecto benéfico de la conducta afectiva infantil sobre el desarrollo de diversos aspectos del comportamiento ha mostrado la importancia de esta conducta en las interacciones tempranas madre-hijo (Carson \& Parke, 1996; Denham, Renwick \& Holt, 1991; Robinson \& Acevedo, 2001; Russ \& Kaugars, 2000-2001; Schneider-Rosen \& Cicchetti, 1984).

Diversos autores han señalado que la interacción adulto-niño es uno de los contextos más importantes en el cual el niño aprende a comportarse afectivamente y promueve la adquisición del lenguaje con fines comunicativos (Bloom, 1998; Saarni, Mumme \& Campos, 1998; Tomasello, 2001).

La relación anterior ha sido documentada empíricamente en diversos estudios (Hann, Osofsky \& Culp, 1996; Howell, 2001; Morissete, Barnard \& Booth, 1995; Nicely, Tamis-LeMonda \& Bornstein, 1999; Slomkowski, Nelson, Dunn \& Plomin, 1992). Por ejemplo, Hann, Osofsky y Culp (1996) correlacionaron el afecto positivo materno y el ajuste en la interacción madre-hijo a los 13 y 20 meses de edad de los niños y mayores progresos en las habilidades lingüísticas y cognoscitivas del menor a los 30 y 44 meses.

Por el contrario, estudios realizados con madres adolescentes muestran que el escaso comportamiento afectivo y mayor cantidad de órdenes, autoritarismo y respuestas menos elaboradas, descriptivas y poco articuladas, se relaciona con un desarrollo cognoscitivo y lingüístico más pobre de los hijos (Levine \& García Coll, 1985; Zeanah, Boris \& Larrieu, 1997; citados en López, Mendieta, Gutiérrez \& Lartigue, 2001).

En la literatura del comportamiento afectivo la importancia atribuida al lenguaje se circunscribe a considerarlo como un factor que posibilita la comunicación del afecto, es decir, la aparición del lenguaje vocal permite la regulación de las emociones, su descripción, discusión y referencia de los afectos propios y de otros. Así, el lenguaje contribuye al desarrollo y expresión de 
la conducta afectiva del infante, sin embargo, también es posible plantearse en qué medida la conducta afectiva en la interacción madre-hijo posibilita un comportamiento de mayor complejidad lingüística por parte del infante.

La pregunta que nos planteamos aquí es si las características afectivas de la interacción madre-hijo están relacionadas con distintos tipos de complejidad lingüística.

La complejidad lingüística a la que hacemos alusión tiene su base conceptual en la propuesta de Ribes (1990) quien enfatiza las propiedades funcionales del lenguaje como un tipo de conducta. El lenguaje puede identificarse en su complejidad según se trasciendan las restricciones espacio-temporales de la situación y se posibilite mediar convencionalmente la conducta propia y la de los demás.

Desde esta perspectiva se ha mostrado la importancia de complementar los estudios que emplean únicamente índices formales del lenguaje, con estudios en los cuales se pueda identificar diferentes competencias lingüísticas (Cortés \& Delgado, 2001).

Para identificar estas diferencias en la competencia lingüística del niño, en este estudio se trabajó con dos situaciones de juego: simulado y libre.

$\mathrm{El}$ juego, en particular el juego simulado, es un contexto en el cual un mayor nivel de desarrollo lingüístico y el afecto es más patente (Campos et al., 1983; Harris, 1989).

Si la interacción madre-hijo que sucede bajo un ambiente afectivo propicio favorece una conducta infantil de mayor complejidad lingüística entonces es probable que las díadas que se comporten con estas características afectivas se desempeñen de manera más efectiva y compleja en situaciones variadas como las que se han señalado.

A diferencia de las diversas propuestas que contemplan emociones positivas y negativas la aplicación de criterios distintos rompe con esta clasificación enfatizando en su lugar la posibilidad variada y amplia de emociones (Campos et al. 2004; Scherer, 2003) o conductas afectivas (Kantor y Smith, 1975) que pueden ser valoradas como facilitadoras u obstaculizadoras en las interacciones del individuo, constituyéndose en conductas precurrentes 0 disposicionales para la realización efectiva de diversas actividades.

A partir de estas consideraciones el objetivo de este estudio es evaluar las diferencias de complejidad lingüística en tareas de juego que exigen competencias diferenciales para los miembros de la díada y analizar si las diferencias en los tipos de juego se relacionan con características afectivas particulares de la díada. Adicionalmente, observar si lo anterior guarda diferencias según la edad del niño.

Para tal fin, cada díada participó en una sesión de juego libre y una de juego simulado. En la primera, la actividad de los participantes no está restringida por ningún criterio impuesto por el experimentador, por el contrario, son ellos 
mismos quienes la estructuran. Esta primera situación refleja la manera que emplea la madre para jugar con su hijo. Suponemos que los estilos de la madre generan desempeños diferenciales del niño y que en situaciones en las cuales ella es la encargada de dirigir la actividad, permitirá establecer la relación entre el comportamiento materno y la complejidad conductual del infante.

A diferencia del juego libre, el juego simulado demanda mayores competencias del niño (Bretherton, Fritz, Zahn-Waxler \& Ridgeway, 1986; Lilard, 1993; López, Etxebarria, Fuentes \& Ortiz, 1999). Este juego se caracteriza por la forma en que se usan los juguetes u objetos, en donde sus propiedades se "transforman" o "alteran" y se usan como si fueran otra cosa o tuvieran vida, recreando situaciones en donde a los juguetes y objetos se le dota de propiedades que no poseen (hablar, sentir, volar) y los usan para fines distintos a los que le son propios (emplear un cubo como carro, mueble o algún aparato).

En esta sesión la responsabilidad de estructurar la actividad es asignada al niño, pretendiendo con esto que se muestre el nivel de competencia del infante en tareas que se consideran más demandantes de las habilidades lingüísticas de los niños.

Así, la comparación de las interacciones en estos dos tipos de juego permitirá identificar si cada contexto se relaciona con diferentes formas de conducta afectiva del niño y de la madre.

La edad, aunque no es un determinante para la evolución conductual del infante comúnmente acompaña diferencias cualitativas y cuantitativas del comportamiento (Rosales \& Baer, 1996). Considerando esto, se seleccionaron díadas con niños de 3 y 5 años de edad dadas las distintas competencias en el juego que muestran los niños en esas edades (Haight, Wang, Fung, Williams \& Mintz, 1999), a fin de identificar diferencias en la conducta afectiva de la díada en función de la mayor independencia del infante en el juego.

\section{MÉTODO}

Diseño

El estudio fue observacional y transversal. Como variables independientes se consideraron las situaciones de juego libre y simulado y el tipo de juguetes empleados en cada uno, familiares (propiedad del niño) y no familiares (provistos por el investigador), respectivamente.

\section{Participantes}

La muestra fue por conveniencia y consistió de 8 niños con sus mamás. Hubo dos niñas y dos niños en cada grupo de edad. Las díadas con niños de 3 años conformaron el grupo G3A (edad promedio de 36.25 meses) y las díadas con niños de 5 años el grupo G5A (edad promedio de 60.9 meses). 
Al momento de la filmación las dos niñas del G5A habían cursado un año de preescolar.

Las características demográficas de las madres de ambos grupos fueron equivalentes en términos de edad ( $X=25.5$ años), ingresos $(X=3$ salarios mínimos) y ocupación (hogar). La escolaridad fue heterogénea e iba desde primaria hasta licenciatura.

\section{Escenario y Materiales}

Las filmaciones se realizaron en el hogar de los participantes con una cámara de video Hi8 marca Handycam Vision Sony Mod. CCD-TRV108 NTC. Al momento de la filmación sólo se encontraban los participantes y la persona que filmaba, quien se ubicó de frente a la díada y a una distancia prudente para permitir el desplazamiento de los participantes.

Los juguetes empleados para el Juego Libre (JL) eran propiedad de los niños y fueron elegidos por ellos y sus mamás.

En el caso de las niñas de ambas edades, los juguetes elegidos fueron muñecas, juegos de té, cuadernos para dibujar o escribir y diversos muñecos de peluche. Los niños eligieron autopistas, carros, muñecos relativos a caricaturas, trompos, soldados, camiones y juegos de mesa. ${ }^{2}$

Para la sesión de Juego Simulado (JS) los juguetes fueron proporcionados por el experimentador y consistieron de diverso muñecos de peluche, un juego de bebés de plástico con sus accesorios, carros miniatura de metal, alimentos de plástico (frutas, verduras, latas y cajas de cereal) y bloques para construcción. Las filmaciones se realizaron entre las 16:00 y 18:00 horas.

\section{Procedimiento}

Cada díada fue filmada en una sesión de JL y una de JS de 30 minutos cada una con un día de separación. En todos los casos primero se realizó el JL y a la mamá se le dieron las siguientes indicaciones:

"Ud. juegue con su hijo(a) de la manera en que normalmente lo hacen y con lo juguetes que elijan. Proponga y dirija el juego, pero no impida que el niño(a) también pueda hacerlo".

La siguiente sesión fue de JS con la siguiente indicación:

"Ahora jugaran con los juguetes que yo les proporcionaré. Pueden inventar historias o situaciones donde los juguetes tengan movimiento y voz, procurando que el niño proponga y dirija el desarrollo del juego, ayudándolo si es necesario o él lo solicita. Codificación.

2. Los juegos de mesa no corresponden a situaciones de juego libre que reporta la literatura, pero uno de los niños de 5 años solo contaba con juegos de esta naturaleza por lo que se procedió a filmar en esta actividad. 
De los videos se codificaron los 20 min. intermedios descartando los extremos, esto para controlar factores de habituación a la cámara y fatiga. Para los datos del nivel funcional se siguieron los criterios empleados por Cortés, Romero y Hernández (2002), y los episodios de simulación y no simulación fueron identificados con base en las siguientes características:

a) Simulado, cuando cualquiera de los participantes atribuyó a los objetos u eventos presentes, a la otra persona o a sí mismo, alguna característica no poseída. Por ejemplo, que el niño, o la madre, finjan que son algún personaje, etc., y,

b) no simulación, cuando los participantes respondían a objetos, eventos o personas de acuerdo a las características o atribuciones que les son propias. Por ejemplo, construcción con los juguetes de ensamble o que los participantes platiquen entre ellos de objetos personas o eventos presentes o ausentes, etc.

Un episodio comenzaba cuando alguno de los participantes se involucraba en alguna situación que cumplía con los criterios señalados y finalizaba cuando alguno de los participantes salía de cuadro, se involucraba en actividades no relacionadas con la que estaba en curso o pasaba a otro juego.

A partir del $25 \%$ de los registros se obtuvo la confiabilidad intra-observador, con 3 meses de separación entre las codificaciones. Se obtuvo un valor Kappa de 0.73 para la complejidad funcional de la madre y de 0.71 para la del niño. Para los episodios de simulación y no simulación los valores Kappa fueron, para la madre de 0.78 y para el niño de 0.79 .

Para la identificación de la conducta afectiva se empleó una taxonomía conductual (Hernández-Castro, 2008) cuyas categorías de la mamá y del niño se muestran en las tablas A1 y A2, respectivamente, del Apéndice A.

Para la codificación de los videos inicialmente se registró el tipo de episodio (simulado o no simulado) y el nivel funcional de los participantes. Posteriormente, se procedió a calificar el tipo de comportamiento afectivo en cada uno de los segmentos funcionales.

Así, de cada participante se obtuvo el tipo y duración del nivel funcional, el tipo y frecuencia de conducta afectiva en cada nivel funcional y el número de episodios de simulación y no simulación.

\section{RESULTADOS}

Para fines de exposición, el análisis de los resultados se presenta como aquellos relativos al nivel funcional y los otros como de conducta afectiva; sin embargo, nuestro punto de partida conceptual es considerar la conducta como funcional. 
Inicialmente se presentan los datos del nivel funcional como proporciones de tiempo para cada miembro de la díada; posteriormente los porcentajes de conducta afectiva en cada tipo de juego, y por último, las relaciones estadísticamente significativas mediante un análisis Log lineal con distribución Poisson. Este análisis se realizó para establecer la relación entre las conductas afectivas con: a) los niveles funcionales de conducta de los niños y las mamás, b) el grupo, y c) el tipo de juego.

\section{Nivel funcional}

Durante ambas condiciones de juego los niños del grupo de 3 años (G3A), y sólo en el juego libre los niños del grupo de 5 años (G5A), interactuaron principalmente en niveles suplementario y selector. En los niños del G3A se observa escasa proporción de tiempo en actividades del tipo sustitutivo referencial que se mantiene en los juegos libre y simulado (Figura 1).
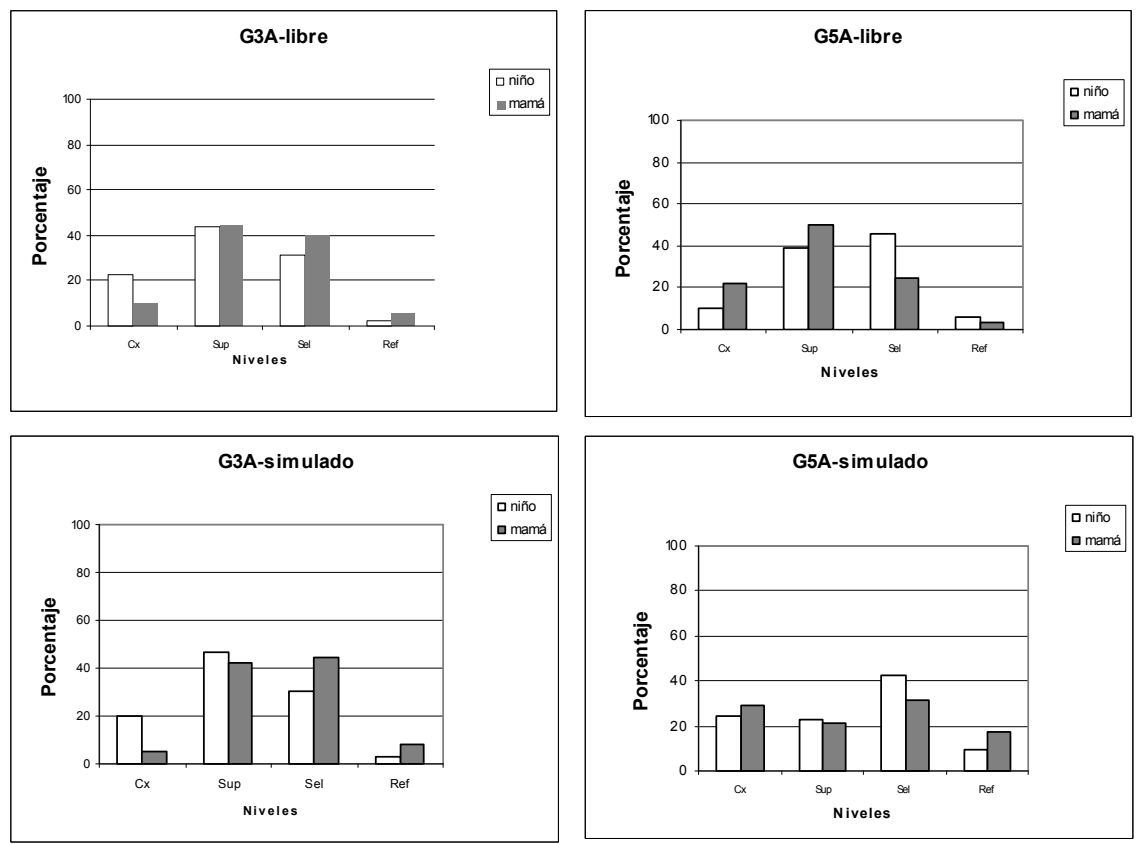

Figura 1. Muestra el porcentaje promedio de tiempo en cada uno de los niveles funcionales en los que transcurre la interacción. La barra obscura representa los datos de la madre y la barra clara los del niño. En la parte superior se presentan los datos en juego libre y en la parte inferior los de juego simulado. En las gráficas que forman la columna izquierda se presentan los datos del G3A y en la columna derecha los del grupo G5A. 
En el caso de los niños del G5A, el tiempo en que su actividad es más compleja presenta cambios de uno a otro juego. En el juego simulado, la conducta selectora sigue siendo prominente y se incrementan los tiempos de conducta sustitutiva referencial.

En el caso de las mamás del G3A, se destaca un patrón semejante en ambas situaciones de juego en las cuales prevalecen las conductas suplementaria y selectora. Sin embargo, su actividad sustitutiva referencial incrementa de $5.26 \%$ en el juego libre a $8.3 \%$ en el simulado. Esto contrasta con la conducta de las mamás del G5A.

Para estas últimas la conducta suplementaria, predominante en el juego libre, se reduce notablemente y se incrementan los otros tipos de comportamiento, es particularmente interesante que la conducta sustitutiva referencial pasa de un $3.8 \%$ de tiempo en el juego libre a $17.6 \%$ en el juego simulado.

En síntesis, nuestros datos indican que las díadas del G3A no se ajustan a los nuevos criterios de juego, sugiriendo que la interacción madre-hijo puede cumplir distintos criterios en tanto el niño es más hábil para estructurar e involucrarse en el juego donde las características de los objetos son sustituidas.

\section{Conducta afectiva}

La Tabla 1 presenta el porcentaje de la conducta afectiva positiva de los niños y las mamás en los tipos de juego.

Tabla 1. Porcentaje de conductas afectivas de las díadas en ambos juegos.

\begin{tabular}{lrrrrrrrrrrr}
\hline & \multicolumn{3}{c}{ Afecto Niños } & & & \multicolumn{9}{c}{ Afecto Mamás } \\
& Obs & $\mathbf{C c}$ & $\mathbf{C f +}$ & $\mathbf{R a}$ & $\mathbf{A i}$ & $\mathbf{A c}$ & $\mathbf{O b s}$ & $\mathbf{C c}$ & $\mathbf{G v +}$ & $\mathbf{C f +}$ & $\mathbf{E x c}$ \\
\hline G3A & & & & & & & & & & & \\
Libre & 52.2 & 8.4 & 0 & 11.2 & 17.4 & 10.7 & 63.7 & 13.8 & 28.4 & 1 & 13.8 \\
Simulado & 52.6 & 11.8 & 2 & 7.2 & 13.2 & 13.2 & 61.4 & 15.3 & 24.2 & 1 & 22.6 \\
G5A & & & & & & & & & & & \\
Libre & 32.3 & 13.4 & 0 & 19 & 22 & 13.4 & 54.9 & 11.8 & 32.3 & 0 & 3 \\
Simulado & 48.7 & 8.1 & 0 & 10.6 & 17.5 & 15 & 61.9 & 10.5 & 15.8 & 0 & 11.8 \\
\hline
\end{tabular}

Nota: Obs=Observación; $\mathrm{Cc}=$ Contacto cara a cara; $\mathrm{Cf}+=$ Contacto físico positivo;

$\mathrm{Ra}=$ Reacciones afectivas; $\mathrm{Ai}=\mathrm{Afectos}$ iniciados; $\mathrm{Ac}=\mathrm{Aceptación.} \mathrm{C} v+=\mathrm{Gestos}$

y vocalizaciones positivas; Exc=Expresiones Cariñosas.

Inicialmente se llevó a cabo un análisis considerando los factores "tipo de juego" y "grupo de edad" y su asociación con cada una de las categorías de afecto. Se encontraron las siguientes relaciones significativas en la situación de juego: para la madre, Gestos y vocalizaciones negativas $(t=3.02, p=.019)$ 
y para el niño Rechazo ( $t=-3.77, p=.007)$. En todos los casos los valores indican un peso mayor para el juego simulado.

En el caso de las relaciones con el Grupo como factor, la única relación significativa para las conductas de la madre fue 'Expresiones cariñosas' a favor del juego libre $(t=3.39, p=0.012)$

Con la finalidad de precisar las relaciones de la conducta afectiva con el nivel funcional, se realizó un segundo análisis en el cual se consideraron los dos grupos y situaciones de juego de manera independiente.

De este segundo análisis, las relaciones estadísticamente significativas se presentan en la Tabla 2. En ésta se puede notar que las asociaciones significativas de conducta afectiva positiva con las dos condiciones de juego, es mayor en el caso del juego libre para ambos participantes. En términos de los grupos, los niños del G5A presentan un mayor número de asociaciones significativas. La descripción detallada de estos datos se presenta enseguida, en conjunto con los porcentajes de las conductas. 


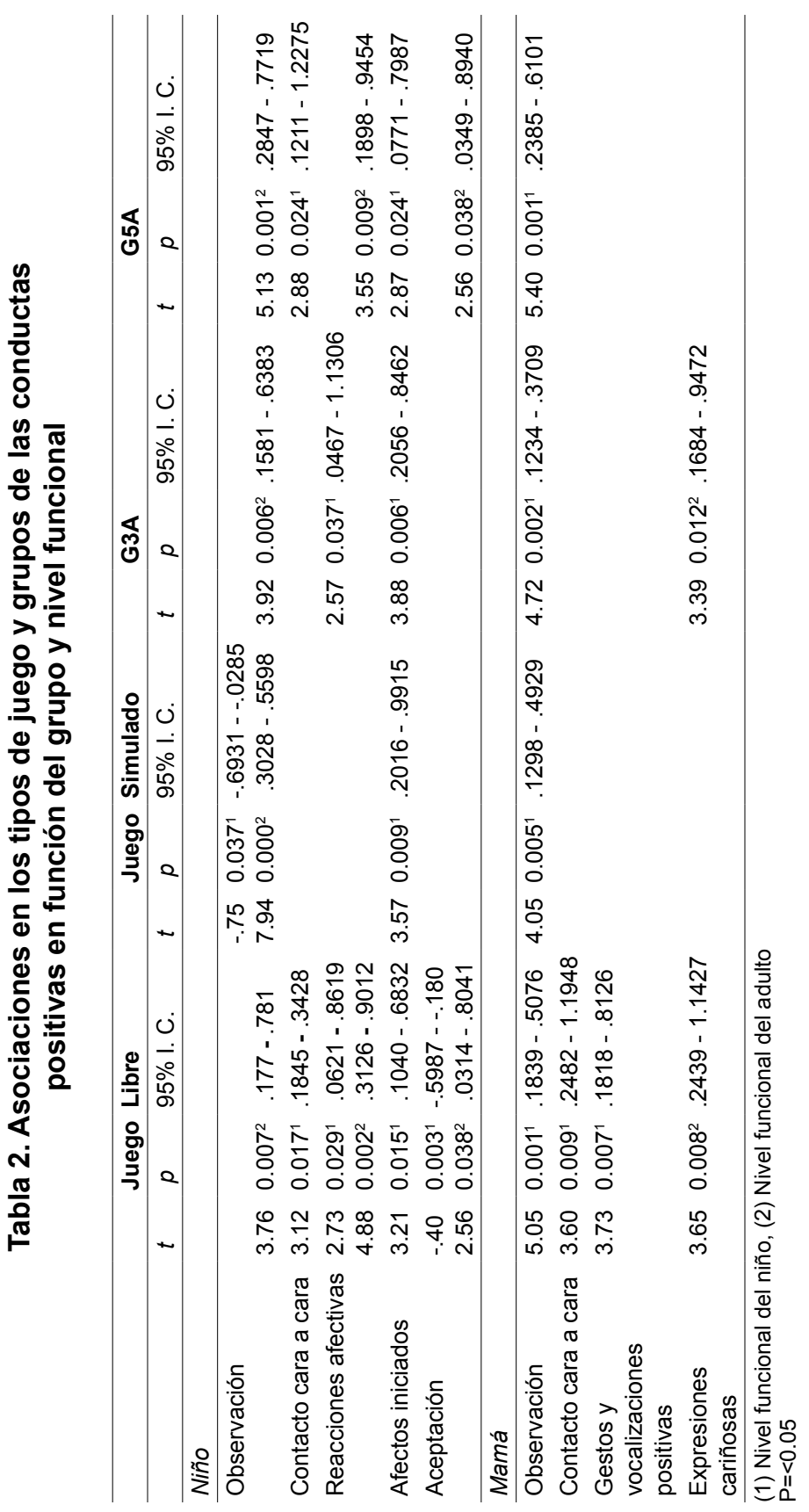


En la Tabla 1 se observa que en ambos grupos la conducta de mayor porcentaje en los dos tipos de juego es la de Afectos Iniciados (excepto en juego simulado del G3A). Del conjunto de conductas la de 'Contacto físico positivo' fue menos frecuente (G3A en juego simulado), o simplemente no se observó.

Del conjunto de conductas positivas se destaca que en ambos grupos la ocurrencia de 'Reacciones afectivas' y 'Afectos iniciado' decrementa de JL a JS, mientras que la de 'Aceptación' presenta un sentido opuesto. Descontando la categoría de 'Contacto físico positivo', todas las conductas se relacionaron con valores moderados ( $p$. ej. 'Reacciones afectivas', $t=2.73, p=0.029$ y 'Aceptación', $t=2.56, p=0.038$ ).

El análisis estadístico indica que, en el caso de los infantes, no existen diferencias entre los tipos de juego ni entre los grupos, más bien es el nivel funcional en que participa cada miembro de la díada el que aumenta la probabilidad de ocurrencia de uno u otro tipo de conducta afectiva.

En el caso de los niños, considerando únicamente el tipo de juego, la misma conducta se asociada con la conducta de ambos participantes. Por ejemplo, la categoría de 'Reacciones afectivas' en la situación de Juego Libre, se asoció a las conductas del niño $(t=2.73, p=0.029)$ y de la madre $(t=4.88, p=0.002)$.

Estos datos replican y coinciden con los porcentajes en la Tabla 1. Adicionalmente, nos indican que este tipo de conducta ocurre con mayor probabilidad en el G5A en función del comportamiento de la madre.

En general, el resultado de los análisis realizados muestra un mayor número de conductas afectivas asociadas con el juego libre respecto del juego simulado, y con el G5A respecto del G3A. En el juego libre se observa que las relaciones significativas están a favor del G3A, excepto la conducta de 'Aceptación' la cual resultó a favor del G5A ( $t=-4.40, p=0.003)$ cuando está asociada al nivel funcional del niño, pero no al del adulto.

Respecto al comportamiento materno en cada uno de los grupos y situaciones, es evidente la escasa o nula ocurrencia de la conducta de "Contacto físico positivo" (Cf+), la cual ocurre en ambos juegos pero sólo en el caso de las mamás del G3A. Estos datos son semejantes en su ocurrencia a los observados por parte de los niños.

Por otra parte, la conducta con mayor ocurrencia es la de 'Gestos y vocalizaciones positivas', que con diferencias de magnitud son las más frecuentes de toda esta clase de conductas. En ambos grupos las mamás presentan una cantidad mayor cuando la situación es de juego libre y son las mamás del G5A las que presentan el porcentaje más alto (28.4 vs. 32.3). Estas conductas decrementan ligeramente para los adultos del G3A (de 28.3 a 24.2), mientras que el decremento es más pronunciado en los adultos del G5A (de 32.3 a 15.8). 
Otra de las conductas donde se observan cambios importantes es en 'Expresiones Cariñosas'. Dos aspectos son los más prominentes: uno, que en los niños del G3A la frecuencia es mayor, y, dos que en ambos grupos es mayor el número de esta conducta en la situación de juego simulado.

Respecto a los contactos cara a cara, las diferencias son pequeñas en ambos grupos, con un ligero incremento de juego libre a simulado para las mamás del G3A y un decremento, igualmente mínimo, en las mamás del G5A.

De las asociaciones significativas encontradas para las categorías maternas (Tabla 2) se destaca que, a excepción de la 'Observación', las demás categorías resultan significativas sólo en el juego libre y su ocurrencia está mayoritariamente relacionada con el nivel funcional del niño. Es importante notar que aun cuando los valores presentados en la Tabla 1 se observa un mayor porcentaje de 'Expresiones cariñosas' en el G5A, esta categoría no resultó significativa en el análisis estadístico realizado. Lo que indica que su ocurrencia no es sistemática respecto de la conducta del compañero de juego, es decir, no guarda relación con algún tipo específico de la conducta del hijo.

\section{DISCUSIÓN}

La pregunta central del presente trabajo era si un comportamiento más complejo de las díadas madre-infante estaba relacionado con el predominio de algún tipo de conducta afectiva.

Se esperaba que la conducta de los niños en la situación de juego simulado, la cual demanda un comportamiento más elaborado, presentara una frecuencia y/o duración mayor de episodios sustitutivos referenciales y una frecuencia mayor del comportamiento afectivo positivo.

El desempeño de los niños del G3A no coincide con los reportados en otros estudios, particularmente el hecho de que los niños pequeños no muestran el cambio esperado en las situaciones que demandan una mayor autonomía de las propiedades físicas de los objetos (vgr. Belsky \& Most, 1981). Esta discrepancia tiene algunas explicaciones posibles.

Primero, es probable que las instrucciones a las madres las hayan inducido a asumir la responsabilidad de que la actividad fuera continua, de tal modo que en la sesión de juego simulado la indicación de "promover que el niño proponga y dirija" pudo no tener mayor relevancia. Sin embargo, esto sólo sería aplicable para las madres del G3A, puesto que en el grupo de niños mayores, el comportamiento de ambos miembros de la díada fue diferente. Así, las instrucciones a las mamás pueden ser descartadas como un factor importante que definiera la conducta del grupo G3A. 
Esto nos obliga a pensar en una segunda explicación la cual tiene que ver con una mayor competencia conductual relacionada con una experiencia más amplia de los niños. Este aspecto puede observarse en la mayor proporción del nivel sustitución referencial en que se involucra el niño. A pesar de que los datos obtenidos respecto a la función de mediador de los niños muestran que aquellos de 5 años estructuran un mayor número de episodios de nivel selector cuando están en la situación de juego simulado respecto del juego libre y un decremento en la frecuencia en los de mayor complejidad. Así, las interacciones más sostenidas inducen a pensar que en este caso la complejidad de la conducta estuvo relacionada con una mayor habilidad y experiencia en el juego.

Adicionalmente, en el caso de los niños del G3A, estos mostraron pocas habilidades para definir las actividades de juego, propiciando que el adulto fuera quien tomara la iniciativa en la organización de la actividad.

Respecto a la relación entre la conducta afectiva y su relación con el desempeño lingüístico, los datos obtenidos no son acordes con los reportados en la literatura. Sin embargo, algunas diferencias entre las distintas investigaciones, permitirá valorar los presentes datos.

La comparación con otros estudios resulta difícil por el tipo de medida realizada para evaluar el lenguaje y el afecto. Los índices de complejidad o desarrollo del lenguaje han sido analizados con base en vocalizaciones y complejidad fonética (vgr. Howell, 2001) y lenguaje expresivo y receptivo (Robinson \& Acevedo, 2001; Tamis-LeMonda, Bornstein \& Baumwell, 2001); mientras que las medidas de lenguaje tomadas en este estudio están más dirigidas a las características funcionales de la interacción, esto es, a la forma relativamente independiente del hacer del infante respecto de las restricciones situacionales.

No obstante que no se observa una relación estrecha entre mayor complejidad lingüística del niño y reacciones afectivas positivas, los datos reportados indican aspectos importantes a ser considerados.

Lo que este dato puede estar señalando es que, por un lado, la naturaleza del juego simulado demanda actividad que puede ser incompatible con el tipo de conducta afectiva que hemos establecido aquí en la medida que se requiere estructurar las actividades en función de criterios explícitos, como es sustituir las propiedades físicas de los objetos mientras que en el juego libre los criterios se cambian constantemente y la interacción no se circunscribe a una planeación.

En concordancia con algunos estudios (De Weerth \& Van Geert, 2002), en la presente investigación la frecuencia de contacto físico fue muy baja, o nula, para los miembros de las díadas de ambos grupos. Este dato es considerado como predecible en díadas normales, bajo la consideración que los 
patrones de conducta sufren cambios en la medida que evoluciona el infante, es decir, el contacto físico materno es más frecuente cuando los niños son de menor edad que los de este estudio; conforme los niños tiene más edad el contacto físico tiende a decrementar en virtud de un mayor desplazamiento y autonomía del infante.

Bajo la consideración de que el afecto es parte de las condiciones para el aprendizaje de un tipo de comportamiento particular, así como un factor que auspicia la resolución de un problema (Barajas \& Clemente, 1999), el desarrollo de actividades en las cuales se deba cumplir con un criterio, sea explícito o implícito, se ve favorecido por las condiciones tanto físicas como interpersonales que implican lo que denominamos conducta afectiva. Así, la variedad de juegos, las representaciones y el intercambio verbal para fines de enseñanza se ven optimizados cuando aparte de las estrategias de enseñanza y los objetos para ello, los interlocutores interactúan en un "ambiente" apropiado para la regulación y retroalimentación mutua. Este proceso puede tener como consecuencia la transferencia de un mejor desempeño del individuo en otras situaciones físicas y sociales, debido a que han estado involucrados en el proceso de instrucción, o de interacción, los elementos necesarios para el aprovechamiento de esas "experiencias".

Así, poder aprender, ejercitar o desarrollar ciertas destrezas, puede estar vinculado no sólo a la aplicación de estrategias de enseñanza formal o informal, sino también a las características afectivas de los que interactúan.

Este primer acercamiento al estudio del comportamiento afectivo en situaciones diádicas contiene tanto logros y ventajas como limitaciones y desventajas.

Una primera ventaja que podemos señalar del presente trabajo es que la evaluación de la conducta afectiva se realiza en situaciones en las cuales se construye la conducta social, es decir, en la interacción con otros individuos (Adamson \& Bakeman, 1985).

El hecho de medir de manera concurrente dos tipos de conducta (complejidad conductual y afecto) permite establecer una relación entre la estructuración y logro de la actividad y los elementos secundarios que rodean la misma. Permite, asimismo, un análisis más fino del modo en que contribuye la conducta afectiva a la organización, desarrollo y culminación de la actividad diádica. La combinación de medidas verbales y no verbales (gestuales) permite establecer la concurrencia de diversos matices de lo que llamamos afecto.

Adicionalmente, la validez ecológica del estudio se incrementa ya que el escenario de observación fue el hogar de la díada, a diferencia de otros estudios en los que las situaciones de juego son en contextos de laboratorio, lo cual puede marcar una diferencia en términos ecológicos (Belsky, 1980). En conjunto con esto, los juguetes con los cuales se realizaron las actividades fueron inicialmente los elegidos por los mismos niños, lo que permite ver de 
una manera más natural la forma en que ellos juegan y cómo se involucra la madre con ellos; permitiendo así, analizar características conductuales de las madres cuando juegan con sus hijos y en qué medida esta forma de interacción contribuye en la sofisticación para el juego del niño realización u obstaculiza para que el niño se comporte de manera más compleja.

Algunas de las limitaciones de nuestro estudio son las siguientes. Primero, los hallazgos no son susceptibles de ser generalizados debido al tamaño de la muestra, el número de sesiones y la edad de los niños. Puesto que las reacciones afectivas son construidas a partir de los contactos del individuo con su entorno físico y/o social, asumimos que la respuesta afectiva del niño hacia objetos y personas está plenamente diferenciada, y que dos únicos registros de su conducta pueden ser insuficientes para poder identificar una relación más íntima entre el afecto y la evolución de la conducta lingüística. Esto lleva a la necesidad de realizar estudios de tipo longitudinal y con niños más pequeños.

Una segunda limitación es la relativa a la situación en la que se realizó la observación. Aunque se ha documentado la importancia del juego, particularmente el juego simulado, como una situación apropiada para la observación del comportamiento afectivo y cognoscitivo; este contexto no es el único en el cual la interacción madre-hijo se desarrolla e impacta al infante (GalbodaLiyanage, Scott \& Prince, 2003).

En consecuencia, la evaluación de esta conducta y su probable relevancia en el desarrollo del lenguaje deberá continuar explorando distintas situaciones de interacción cuidador-niño a partir de edades más tempranas del infante.

\section{REFERENCIAS}

Adamson, L. B. \& Bakeman, R. (1985). Affect and attention: Infant observed with mothers and peers. Child Development, 56, 582-593.

Barajas, C. \& Clemente, R. A. (1999). El apoyo materno a la solución de problemas. Propuesta de un sistema de clasificación. En M. T. Anguera (Coord.) Observación de conducta interactiva en contextos naturales: aplicaciones. 189-222, Barcelona, España: Edicions Universitat deBarcelona.

Belsky, J. (1980). Mother-infant interaction at home and in the laboratory: A comparative study. The Journal of Genetic Psychology, 137, 37-47.

Belsky, J. \& Most, R. (1981). From exploration to play: A cross-sectional study of infant free play behavior. Developmental Psychology, 17, 630-639.

Bloom, L. (1998). Language acquisition in its developmental context. En W. Damon (Ed. de la serie) \& R. M. Lerner (Ed. del volumen), Handbook of Child Psychology: Vol. 2. Cognition, perception, and language (5a. ed., pp. 309-370). New York: Wiley. 
Bretherton, I., Fritz, J., Zahn-Waxler, C. \& Ridgeway, D. (1986). Learning to talk about emotions: A functionalist perspective. Child Development, 57, 529-548.

Campos, J. J., Barret, K. C., Lamb, M. E., Goldsmith, H. H. \& Stenberg, C. (1983). Socioemotional development. En P. H. Mussen (Ed. de la serie) \& M. Haith \& J.

Campos (Eds. del volumen), Handbook of Child Development: Vol. 2. Infancy anddevelopmental psychobiology (4a. ed., pp. 435-571). New York: Wiley.

Campos, J. J., Frankel, C. B. \& Camras, L. (2004). On the nature of emotion regulation. Child Development, 75(2), 377-394.

Carson, J. L. \& Parke, R. D. (1996). Reciprocal negative affect in parent-child interactions and children's peer competency. Child Development, 67, 2217-2226.

Cortés, A. \& Delgado, U. (2001). Análisis funcional del desarrollo lingüístico. En: G. Mares \& Y. Guevara Psicología Interconductual. Avances en la Investigación Básica, México: UNAM-FES Iztacala.

Cortés, A., Romero, P. \& Hernández, R. (2002). Interacciones niño-niño en tareas de simulación: la importancia de la historia de mediación. Universitas Psychologica, 1(1), 7-18.

Denham, S. A., Renwick, S. M. \& Holt, R. W. (1991). Working and playing together: Prediction of preschool social-emotional competence from mother-child interaction. Child Development, 62, 242-249.

De Weerth, C. \& Van Geert, P. (2002). Changing patterns of infant behavior and mother-infant interaction: Intra -and interindividual variability. Infant Behavior \& Development, 24, 347-371.

Galboda-Liyanage, K. C., Scott, S. \& Prince, M. J. (2003). Time budgets of the mothers of pre-school children: An analysis of mother-child joint activities. British Journal of Development Psychology, 21, 273-283.

Haight, W., Wang, X., Fung, H., Williams, K. \& Mintz, J. (1999). Developmental, and variable aspects of young children's play: A cross-cultural comparison of pretending at home. Child Development, 70(6), 1477-1488.

Hann, D. M., Osofsky, J. D. \& Culp, A. M. (1996). Relating the adolescent motherchild relationship to preschool outcomes. Infant Mental Health Journal, 17(4), 302-309.

Harris, P. L. (1989). Children and emotion: The development of psychological understanding. Oxford: Basil Blackwell.

Hernández-Castro, R (2008). El comportamiento afectivo en las interacciones madrehijo: su contribución al desarrollo del lenguaje. Tesis de maestría no publicada, UNAM, Facultad de Estudios Superiores Iztacala: México.

Howell, C. S. (2001). Mothers' speech with 12-month-old infants: Influences on the amount and complexity of infants' vocalizations. Dissertation Abstracts International, 61, 12-A (University Microfilms No. 2001-95011-164).

Kantor, J. R: \& Smith, N. W. (1975). The science of psychology: An interbehavioral survey. Chicago: The Principia Press.

Lilard, A. S. (1993). Pretend play skills and the child's theory of mind. Chile Development, 64, 348-371.

López, F.; Etxebarria, I.; Fuentes, M. J. \& Ortiz, M. J. (1999). (Coordinadores). Desarrollo afectivo y social. Madrid, España: Ediciones Pirámide. 
López, F., Mendieta, A., Gutiérrez, M y Lartigue, T. (2001). Patrones de interacción y apego madre adolescente-hijo(a) desde una metodología observacional. En N. Reyes de Polanco (Ed.) Observación de bebés, México: Asociación Psicoanalítica Mexicana- Plaza y Valdez.

Morisset, C.E., Bernard, K. E. \& Booth, C. L. (1995). Toddlers' language development: Sex differences within social risk. Developmental Psychology, 31(5), 851-865.

Nicely, P., Tamis-LeMonda, C. S. \& Bornstein. M. H. (1999). Mothers' attuned responses to infant affect expressivity promote earlier achievement of language milestone. Infant Behavior \& Development, 22(4), 557-568.

Reissland, N., Shepherd, J. \& Herrera, E. (2003). The pitch of maternal voice: A comparison of mothers suffering from depressed mood and non-depressed mothers reading books to their infants. Journal of Child Psychology and Psychiatry, 44(2), 255-261.

Ribes, E. (1990). El lenguaje como conducta: Mediación funcional versus descripción morfológica. En E. Ribes (Ed.). Psicología General, México: Trillas.

Robinson, J. L. \& Acevedo, M. (2001). Infant reactivity and reliance on mother during emotion challenges: Prediction of cognition and language skills in a low-income samples. Child Development, 72(2), 402-415.

Rosales, J. \& Baer, D. M. (1996). Un punto de vista analítico-conductual del desarroIlo. En S. W. Bijou \& E. Ribes (coordinadores), El desarrollo del comportamiento (pp. 203-242). Guadalajara, México: Universidad de Guadalajara.

Russ, S. W. \& Kaugars, A. S. (2000-2001). Emotion in children's play and creative problem solving. Creativity Research Journal, 13(2), 211-219.

Saarni, C., Mumme, D. L. \& Campos, J. (1998). Emotional development: action, communication, and understanding. En W. Damon (Ed. de la serie) \& N. Eisenberg (Ed. del volumen), Handbook of Child Psychology: Vol. 3. Social, Emotional, and Personality Development (5a. ed., pp. 237-309).New York: Wiley.

Scherer, K. R. (2003). Vocal communication of emotion: A review of research paradigms. Speech Communication, 40(1-2), 227-256.

Schneider-Rosen, K. \& Cicchetti, D. (1984). The relationship between affect and cognition in maltreated infants: Quality of attachment and the development of visual self-recognition. Child Development, 55, 648-658.

Slomkowski, Ch. L.; Nelson, K.; Dunn, J. \& Plomin, R. (1992). Temperament and language: Relations from toddlerhood to middle Childhood. Developmental Psychology, 28(6), 1090-1095.

Tamis-LeMonda, C., Bornstein, M. H. \& Baumwell, L. (2001). Maternal responsiveness and children's achievement of language milestones. Child Development, 72(3), 748-767.

Tomasello, M. (2001). Perceiving intentions and learning words in the second year of life. En M. Bowerman \& S. C. Levinson (Eds.). Language acquisition and conceptual development (pp. 132-158). Cambridge, UK: Cambridge University Press. 


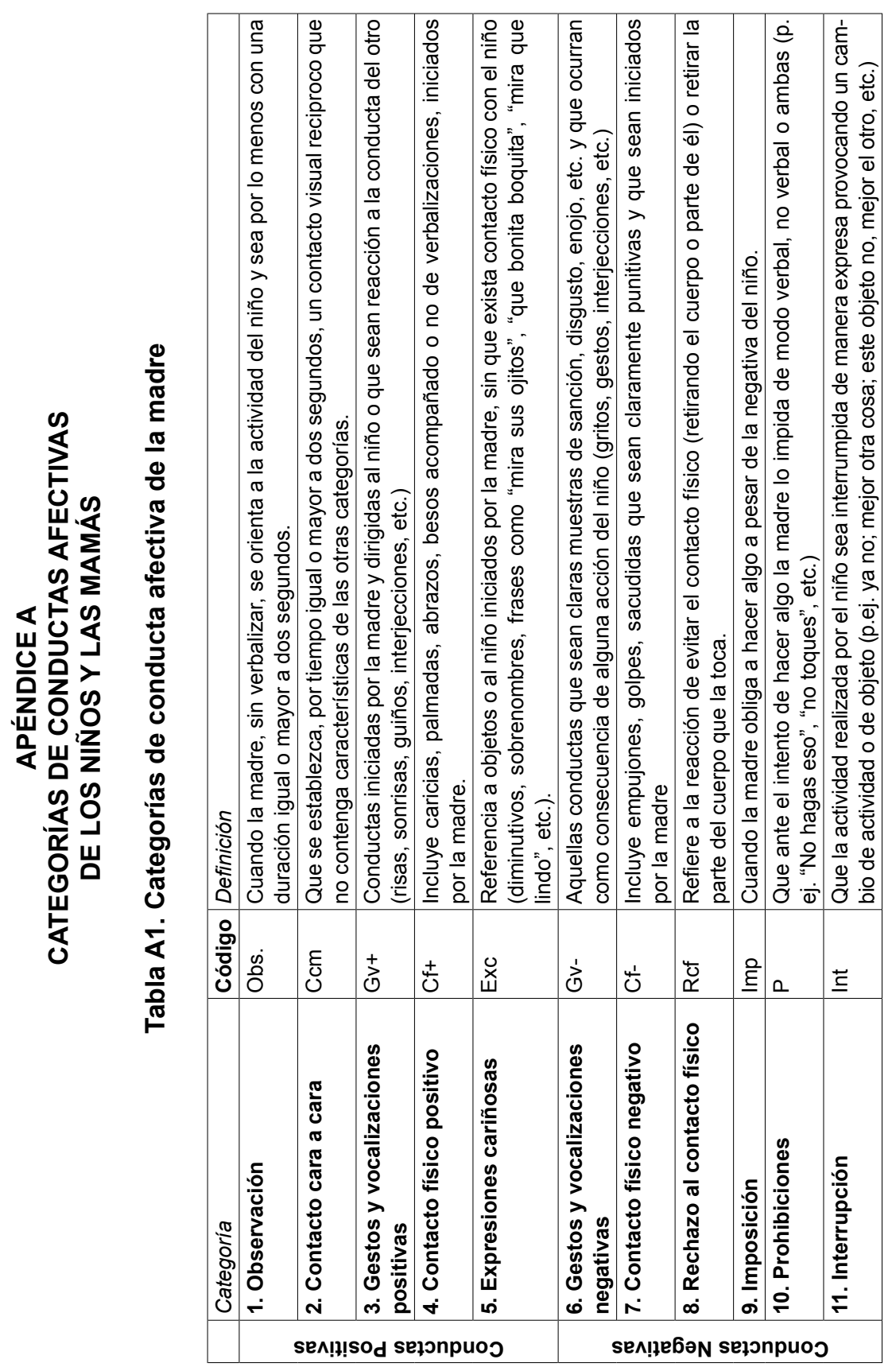




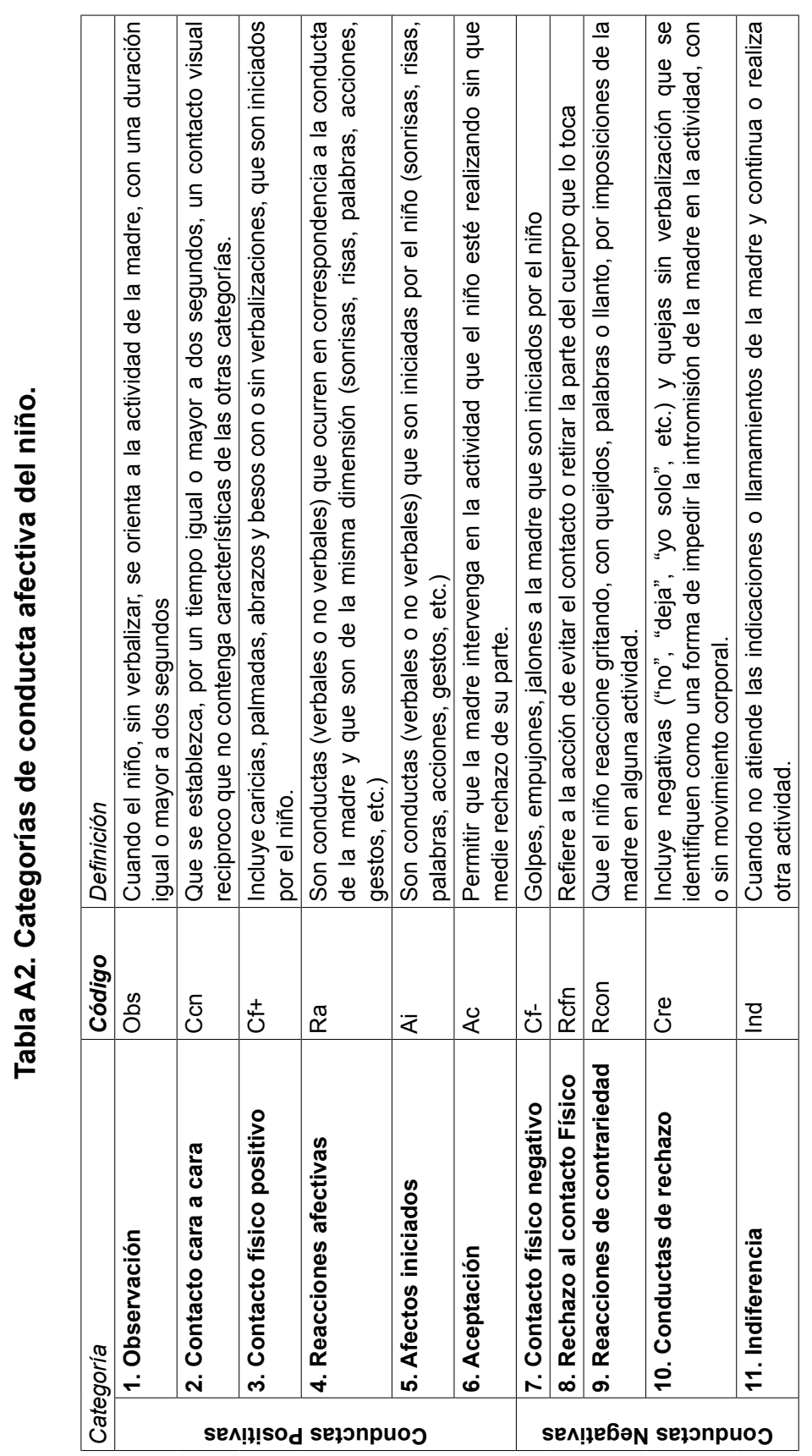

